\title{
Observability analysis and state estimator proposal for the chocolate conching process
}

\author{
$1^{\text {st }}$ Camila Gonzalez Arango \\ OPTIMO, Optimización Matemática de Procesos \\ Universidad Pontificia Bolivariana UPB \\ Medellin, Colombia \\ camila.gonzalez@upb.edu.co
}

$4^{\text {th }}$ Carlos Ocampo-Martinez

Institut de Robòtica I Informàtica Industrial (CSIC-UPC)

Universitat Politècnica de Catalunya

Barcelona, Spain

carlos.ocampo@upc.edu

\author{
$2^{\text {nd }}$ Elly V. Acosta, $3^{\text {th }}$ Juan Camilo Mazo Rivas \\ Centro de Investigación de Desarrollo y Calidad-CIDCA, \\ Compañía Nacional de Chocolates S.A.S \\ Rionegro, Colombia \\ eacosta@chocolates.com.co; jcmazo@chocolates.com.co
}

\author{
$5^{\text {th }}$ Diego A. Muñoz \\ OPTIMO, Optimización Matemática de Procesos \\ Universidad Pontificia Bolivariana UPB \\ Medellin, Colombia \\ diego.munoz@upb.edu.co
}

\begin{abstract}
The conching process is a crucial stage involving the development of sensory attributes and rheological properties of the final product. Currently, the monitoring of critical variables during this process is done off-line through extensive measurements with conventional methodologies based on laboratory analysis, e.g., the quantification of the concentration of the volatile active compounds of the flavor and aroma of a chocolate batch and its viscosity. All of them are related to an indicator for the chocolate sensory quality known as Conching Degree (CD). In this work, the observability analysis for a Phenomenological Based Model for the conching process is done as a first condition for the solution of a state estimation problem. Besides, the initial idea of a virtual sensor is proposed considering the available measurements from the conching process.
\end{abstract}

Index Terms-Phenomenological Based Semi-Physical Model, virtual sensor, state estimation, conching process, chocolate, Conching Degree (CD).

\section{INTRODUCTION}

The conching process is considered as the final stage or the final operation in the chocolate manufacturing, where the chocolate mass is changed from a flaky, dry powder into a fine flowing melt that has an intensive, harmonious and long-lasting flavor [1, 2]. Danzl and Ziegleder [3] proposed the use of a quality sensory indicator of chocolate namely Conching Degree (CD). The CD relates the presence of two volatile compounds, which are chosen as flavor markers of chocolate, namely tetrametilpirazine (TMP) and benzaldehide (BA). A high CD means, in sensory terms, a harmonious and intense chocolate flavor; in rheological terms, proper flow properties and a fine consistency, whilst in chemical terms, an analytically measurable flavor homogenization.

Standardize the conching process is an important objective since it allows defining different strategies to control the variability of raw materials such as cocoa beans. However, there are factors from the harvest and from the required supply needs at each year period that limit the possibility of establishing strictly equal initial conditions. Additionally, during the conching it is important to monitor different chemical, physical and rheological process variables for the final determination of the product quality. Some of the process variables are currently controlled in the PLC of the conches, however, some others, such as the $\mathrm{CD}$, require the quantification of TMP and BA, which are impossible to determine in real time with existing tools since their quantification requires conventional laboratory methods as Gas Chromatography-Mass Spectrometry (GC/MS) $[4,5]$.

On the other hand, the most important effect of the physical changes in the mass rheology is to develop suitable flow properties as a prerequisite for optimum performance regarding flavor perception, and product pleasant mouth feel [1]. Therefore, monitoring the chocolate viscosity is also an essential task for guaranteeing the optimum performance of the product.

For the previous discussion, having this response through the development of Phenomenological Based Semi-Physical Model (PBSM) as virtual sensors would be a convincing result to establish the optimal process times, since it allows the estimation of the $\mathrm{CD}$ and the chocolate's viscosity, by means of the relationship of these variables with other process variables that are possible to quantify in real time, fact that guarantees permanent monitoring of the process, facilitates decision making, and generates improvement opportunities in it.

According to the current production measurements technology for industrial chocolate processes, there are several online and off-line sensors for recording and measuring some typical variables. In that sense, to measure product quality related to the aroma released in the conching process, Tan and Kerr $[6,7]$ use gas sensors as electronic nose system 
installed in a cocoa grinder to predict the overall volatile compounds at different refining stages. The degree of roasting of cocoa beans could be determined as an essential variable in chocolate's quality by training an Artificial Neural Network. On the other hand, Tan and Balasubramanian [8] put forward three suitable alternatives to measure the size of cocoa particles at different stages of refining and conching processes in both conical and cylindrical cocoa grinder configurations. The use of micrometer and light microscopy image analysis are adequate to monitor cocoa's particle size during the processes from the proposed alternatives.

In this work, a PBSM for the conching process, which was previously reported $[9,10]$, will be use with the aim of verifying observability as a first condition for the solution of a state estimation problem. In addition, an initial proposal for the estimation of both the CD and the viscosity of a chocolate batch is addressed and some preliminary results are presented.

The work is organized as follows: in Section II, the general structure for the PBSM of the conching process is reported. In Section III the observability analysis is development, while in Section IV, the preliminary idea for the virtual sensor towards estimating the $\mathrm{CD}$ and the viscosity in the conching process taking into account the available measurements in the process is presented. Finally, some conclusions are drawn in Section $\mathrm{V}$.

\section{Mathematical Model for the CONCHING PROCESS}

To perform state estimation, the observations with explicit physical insight in a mathematical model is a necessary task [11]. In that sense, the Phenomenological Based models for predicting the $C D$ of chocolate [9] and the chocolate's structural changes during conching process [10] will be used to verify the observability. These models allow to follow the evolution of the $C D$ as a chocolate's quality indicator and the dynamic evolution of chocolate's rheological variables. Mathematically, the time-varying $C D$ is computed as

$$
C D=\frac{1}{w_{T M P}+w_{B A}},
$$

where $w_{T M P}$ and $w_{B A}$ are the concentration of TMP and BA, respectively.

The conching process has been divided into three phases: dry, plastic, and liquid. During the dry stage, there is a considerable decrease in the water and the concentration of undesirable volatile compounds while the perception of the desirable ones is enhanced $[1,12]$. During the plastic stage, there are important changes in the mass's rheology; the fat migrates to the surface of the solids, allowing the viscosity reduction of the mass. Furthermore, in the liquid stage, there is an addition of cocoa butter and/or emulsifier promoting mixing and ending the development of the required flow properties [1]. Figure 1 shows the block diagram representation of the Process Systems $P S$ used to develop the considered mathematical model $[9,10]$. Mass and energy balances were performed on each $P S$. Based on the proposed balances, the basic

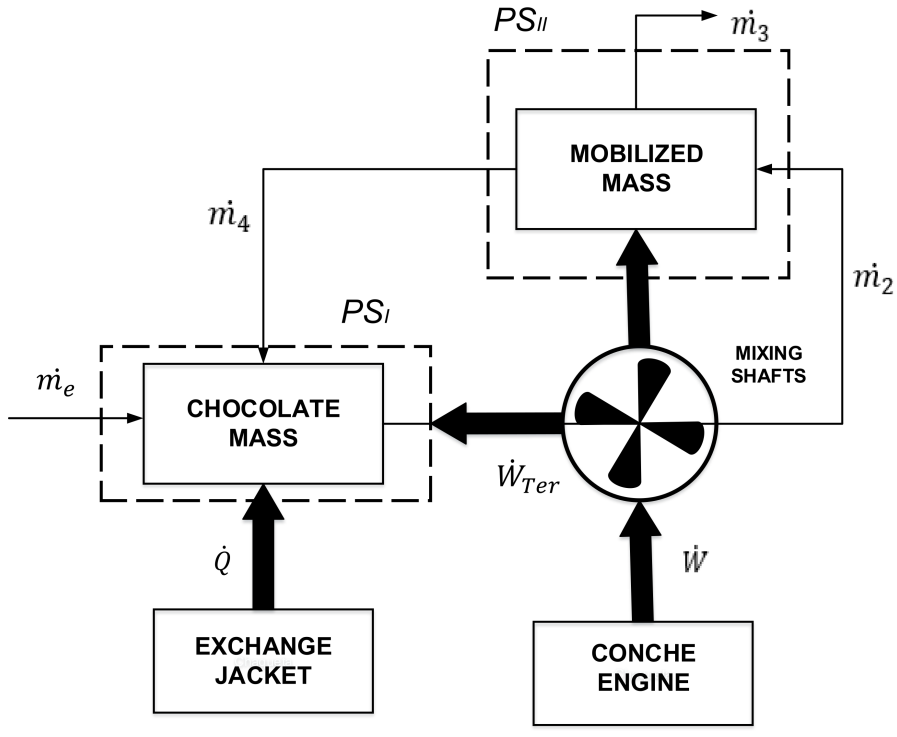

Fig. 1. Process systems defined to model the conching process

model structure consists of 10 differential equations, which are presented below. Variables definition are stated in Tables I and II.

$P S_{I}$ : Mass balances are formulated for water, the volatile compounds (TMP and BA), exposed fat (cocoa butter, CB), and the total mass balance is also considered.

- Total mass balance:

$$
\frac{d}{d t}\left(M_{P S_{I}}\right)=\dot{m}_{e}+\dot{m}_{4}-\dot{m}_{2},
$$

where $M_{P S_{I}}$ is the total mass in $P S_{I}$.

- Mass balance for the moisture content:

$$
\frac{d}{d t}\left(M_{P_{S_{I}}} w_{H_{2} O, 2}\right)=\dot{m}_{e} w_{H_{2} O, e}+\dot{m}_{4} w_{H_{2} O, 4}-\dot{m}_{2} w_{H_{2} O, 2},
$$

where $w_{\mathrm{H}_{2} \mathrm{O}, i}$ is the water concentration in stream $i \in$ $\{2,4, e\}$.

- Mass balance for BA:

$$
\frac{d}{d t}\left(M_{P S_{I}} w_{B A, 2}\right)=\dot{m}_{e} w_{B A, e}+\dot{m}_{4} w_{B A, 4}-\dot{m}_{2} w_{B A, 2} \text {, }
$$

where $w_{B A, i}$ is the BA concentration in stream $i \in$ $\{2,4, e\}$.

- Mass balance for TMP:

$$
\frac{d}{d t}\left(M_{P S_{I}} w_{T M P, 2}\right)=\dot{m}_{e} w_{T M P, e}+\dot{m}_{4} w_{T M P, 4}-\dot{m}_{2} w_{T M P, 2},
$$

where $w_{T M P, i}$ is the TMP concentration in stream $i \in$ $\{2,4, e\}$.

- Mass balance for exposed fat fraction: 
TABLE I

CONSTITUTIVE EQUATIONS FOR THE STRUCTURAL PARAMETERS

\begin{tabular}{|c|c|c|}
\hline \# & Description & Constitutive equation \\
\hline 1 & Mass flow in $\dot{m}_{2}\left[\frac{\mathrm{kg}_{\mathrm{DS}}}{\mathrm{min}}\right.$ & $\dot{m}_{2}=\omega_{s} V_{P S_{I I}} \rho_{c} w_{s o l, 0}$ \\
\hline 2 & Mass flow $\dot{m}_{4}\left[\frac{\mathrm{kg}_{\mathrm{SS}}}{\mathrm{min}}\right]$ & $\dot{m}_{4}=\dot{m}_{2}$ \\
\hline 3 & Mass flow $\dot{m}_{3}\left[\frac{\mathrm{kg}_{\text {mix }}}{\text { min }}\right]$ & $\dot{m}_{3}=\dot{m}_{H_{2} O, 3}+\dot{m}_{T M P, 3}$ \\
\hline 4 & $\begin{array}{l}\mathrm{H}_{2} \mathrm{O} \text { concentration in } \dot{m}_{3} \\
{\left[\frac{\mathrm{kg}_{\mathrm{H}_{2} \mathrm{O}}}{\mathrm{kg}_{\operatorname{mix}}}\right]}\end{array}$ & $\begin{array}{c}+\dot{m}_{B A, 3} \\
w_{H_{2} O, 3}=\frac{\dot{m}_{H_{2} O, 3}}{\dot{m}_{3}}\end{array}$ \\
\hline 5 & $\begin{array}{l}B A \text { concentration in } \dot{m}_{3} \\
{\left[\frac{\mathrm{mg}_{B A}}{\mathrm{~kg}_{\text {mix }}}\right]}\end{array}$ & $w_{B A, 3}=\frac{\dot{m}_{B A, 3}}{\dot{m}_{3}}$ \\
\hline 6 & $\begin{array}{l}T M P \text { concentration in } \\
\dot{m}_{3}\left[\frac{\operatorname{mg}_{T M P}}{\operatorname{kg}_{m i x}}\right]\end{array}$ & $w_{T M P, 3}=1-w_{B A, 3}-w_{H_{2} O, 3}$ \\
\hline 7 & $\begin{array}{l}\text { Stream } 2 \text { specific enthalpy } \\
{[\mathrm{J} / \mathrm{kg}]}\end{array}$ & $\widehat{H}_{2}=C_{p}\left(T-T_{r e f}\right)$ \\
\hline 8 & $\begin{array}{l}\text { Stream } 4 \text { specific enthalpy } \\
{[\mathrm{J} / \mathrm{kg}]}\end{array}$ & $\widehat{H}_{4}=C_{p}\left(T-T_{r e f}\right)$ \\
\hline 9 & Total Heat $[\mathrm{J} / \mathrm{min}]$ & $Q=U A\left(T-T_{j a c k}\right)$ \\
\hline 10 & Electric power $[\mathrm{J} / \mathrm{min}]$ & $\dot{W}=\eta_{m} P_{m}$ \\
\hline 11 & Thermal work $[\mathrm{J} / \mathrm{min}]$ & $\dot{W}_{T e r}=\mu_{J T}^{*} \dot{W}$ \\
\hline 12 & Friction loss $\left[\mathrm{m}^{2} / \mathrm{s}^{2}\right]$ & $h_{f}=K_{F r} \underline{\left(r \omega_{s}\right)^{2}}$ \\
\hline 13 & $\begin{array}{l}\text { Pumping } \\
{\left[\mathrm{kg}_{\mathrm{T}} / \mathrm{min}\right]}\end{array}$ & $P_{c}=N_{Q} w_{s} r_{b}^{3}$ \\
\hline
\end{tabular}

$\frac{d}{d t}\left(M_{P S_{I}} w_{e f}\right)=\rho_{c} \varphi_{C B} P_{C}\left(w_{e f}-w_{a f} k_{r}\right)+\dot{m}_{C B_{e}}$,

where $w_{\text {ef }}$ corresponds to the fraction of the exposed fat with the cocoa butter (CB) already homogenized on the chocolate particles.

- Thermal energy balance

$$
\frac{d}{d t}\left(M_{P S_{I}} C_{p} T\right)=\dot{m}_{4} \widehat{H}_{4}-\dot{m}_{2} \widehat{H}_{2}+\dot{Q}-\dot{W}_{T e r},
$$

where $T$ is the chocolate mass temperature.

- Mechanical energy balance

$$
\frac{d}{d t}\left(\frac{M_{P S_{I}}\left(r_{b} \omega_{s}\right)^{2}}{2}\right)=\dot{W}-\dot{m}_{4} h_{f},
$$

where $\omega_{s}$ is the shaft's radial velocity.

$P S_{I I}$ : The considered balances assumed that there is no mass accumulation in the control volume of this process system, i.e., the mass of DS mobilized $\left(M_{P S_{I I}}\right)$ remains constant.

- Total mass balance:

$$
0=\dot{m}_{2}-\dot{m}_{4}-\dot{m}_{3}
$$

- Mass balance for the moisture content:

$$
\frac{d}{d t}\left(M_{P S_{I I}} w_{H_{2} O, 4}\right)=\dot{m}_{2} w_{H_{2} O, 2}-\dot{m}_{3} w_{H_{2} O, 3}-\dot{m}
$$

where $M_{P S_{I I}}$ is the total mass in $P S_{I I}$.

\begin{tabular}{|c|c|c|}
\hline \# & Description & Constitutive equation \\
\hline 1 & Volume of $P S_{I I}\left[\mathrm{~m}^{3}\right]$ & $V_{P S_{I I}}=\frac{1}{3} \pi r_{b}^{2} L_{b}$ \\
\hline 2 & $\begin{array}{l}\text { Mass flow of } \mathrm{H}_{2} \mathrm{O} \\
{\left[\frac{\mathrm{kg}_{\text {mix }}}{\min }\right]}\end{array}$ & $\dot{m}_{H 2_{O}, 3}=$ \\
\hline & & $\begin{array}{c}K_{\mathrm{O}_{\mathrm{H}_{2} \mathrm{O}}} A_{M}\left(w_{\mathrm{H}_{2} \mathrm{O}, 4}-k_{y} y^{*}\right) \\
V_{P S_{I I}}\end{array}$ \\
\hline 3 & Mass transfer area $\left[m^{2}\right]$ & $A_{M}=\epsilon A_{s p} \frac{P M}{V_{p}}$ \\
\hline 4 & $\begin{array}{l}\text { Superficial area of spheri- } \\
\text { cal particle }\left[\mathrm{m}^{2}\right]\end{array}$ & $A_{s p}=\pi D_{p}^{2}$ \\
\hline 5 & Particle volume $\left[\mathrm{m}^{3}\right]$ & $V_{p}=\frac{1}{6} \pi D_{p}^{3}$ \\
\hline 6 & $\begin{array}{l}\text { Fictional } \\
{\left[\frac{\mathrm{kgH}_{2} \mathrm{O}}{\mathrm{kg}_{\mathrm{DA}}}\right] \text { concentration }}\end{array}$ & $y^{*}=\frac{P_{v}}{\left(P-P_{v}\right)} * \frac{18.02}{28.97}$ \\
\hline 7 & $\begin{array}{l}\text { Exposed particle fraction } \\
{[-]}\end{array}$ & $\epsilon=1-\frac{w_{e f}}{w_{a f_{T}}}$ \\
\hline 8 & Vapor pressure $[\mathrm{Pa}]$ & $P_{v}=1000 \exp \left(16.5362-\frac{3985.44}{T-38.9944}\right)$ \\
\hline 9 & $\begin{array}{l}B A \text { mass flow in } \dot{m}_{3} \\
{\left[\frac{\mathrm{kg}}{\mathrm{min}}\right]}\end{array}$ & $\dot{m}_{B A, 3}=k_{\tau, B A} \dot{m}_{H_{2} O, 3}$ \\
\hline 10 & $\begin{array}{l}\stackrel{T}{T} M P \text { mass flow in } \dot{m}_{3} \\
{\left[\frac{\mathrm{kg}}{\mathrm{min}}\right]}\end{array}$ & $\dot{m}_{T M P, 3}=k_{\tau, T M P} \dot{m}_{H_{2} O, 3}$ \\
\hline 11 & $\begin{array}{l}B A \text { Mass transfer coeffi- } \\
\text { cient }\end{array}$ & $k_{\tau, B A}=\alpha_{B A} \exp \left(-\frac{\beta_{B A}}{\epsilon}\right)$ \\
\hline 12 & $\begin{array}{l}T M P \text { Mass transfer coef- } \\
\text { ficient }\end{array}$ & $k_{\tau, T M P}=\alpha_{T M P} \exp \left(-\frac{\beta_{T M P}}{\epsilon}\right)$ \\
\hline 13 & Friction loss factor $[-]$ & $K_{F r}=f_{D} \frac{L_{T}}{D_{T}}$ \\
\hline 14 & Darcy factor $[-]$ & $f_{D}={\frac{0.3164}{N_{R e}^{0.25}}}^{T}$ \\
\hline 15 & Reynolds number $[-]$ & $N_{R e}=\frac{\rho_{c}\left(r_{b} \omega_{s}\right) D_{T}}{u}$ \\
\hline 16 & $\begin{array}{l}\text { Chocolate viscosity }[\mathrm{Pa}- \\
\mathrm{s}]\end{array}$ & $\mu=\frac{\sigma}{\gamma}$ \\
\hline 17 & $\begin{array}{l}\text { Herschel Bulkley shear } \\
\text { stress }[\mathrm{Pa}]\end{array}$ & $\sigma=\sigma_{o}+k \gamma^{n}$ \\
\hline 18 & Shear rate $[1 / \mathrm{s}]$ & $\gamma=\frac{P_{m}}{V_{P S_{r}} \sigma}$ \\
\hline 19 & Motor Power $[\mathrm{J} / \mathrm{min}]$ & $P_{m}=V I$ \\
\hline
\end{tabular}

- Mass balance for BA:
TABLE II

CONSTITUTIVE EQUATIONS FOR THE FUNCTIONAL PARAMETERS

- Mass balance for TMP:

$$
\begin{aligned}
\frac{d}{d t}\left(M_{P S_{I I}} w_{T M P, 4}\right)= & \dot{m}_{2} w_{T M P, 2}-\dot{m}_{3} w_{T M P, 3} \\
& -\dot{m}_{4} w_{T M P, 4} .
\end{aligned}
$$

Moreover, to have a consistent problem, 13 constitutive equations were defined to explain the structural parameters, and 19 constitutive equations are used to explain the structural and functional parameters of the model, summarized in Tables I and II, respectively. Finally, nine parameters were identified from experimental data. The details of the model construction $4 \mathrm{ean}$ be found in $[9,10]$.

The resulting model corresponds to an index 1 semiexplicit Differential-Algebraic Equations (DAEs) system.Thus, through one differentiation step of the algebraic part of the 
model with respect to time $t$, it is possible to transform the DAEs into an Ordinary Differential Equations (ODEs) system.

Therefore, the resulting ODEs system can be compactly expressed as follows

$$
\dot{x}_{a}=F_{a}\left(x_{a}, u\right),
$$

where the state vector of the system corresponds to $x_{a}=$ $\left[M_{P S_{I}}, w_{H_{2} O, j}, w_{B A, j} w_{T M P, j}, w_{e f}, T, \omega_{s}, \dot{m}_{4}, Q, P_{c}, N_{R e}\right.$, $\mu, \sigma, \gamma]^{T}$.

\section{OBSERVABILITY ANALYSIS}

The observability analysis of a model consists of knowing the conditions that allow to reconstruct the state $x(t)$ from the knowledge of the inputs $u(t)$ and outputs $y(t)$ in a defined interval of time.

Therefore, considering the conching process as a batch process, local observability is verified in a set of finite points of the system's nominal trajectory. To perform observability analysis, it is necessary to know the function that describes the output of the system $y=h\left(x_{a}, u\right)$. To this aim the analysis of the available measurements in the process is presented below.

\section{A. Definition of the system's output function}

Figure 2 shows the location of the temperature and relative moisture sensors which are installed in the conche. Sensor 1 is located in the upper conche compartment, measuring the temperature and relative moisture inside this compartment. Sensor 2 is located outside the conche, measuring the temperature and relative moisture of the conche's surrounding air. In addition, chocolate temperature $T$ is measured by an already installed sensor in the equipment. All of the sensors send a wireless signal to the signal receptor located near of a computer in which the signal is received in real time.

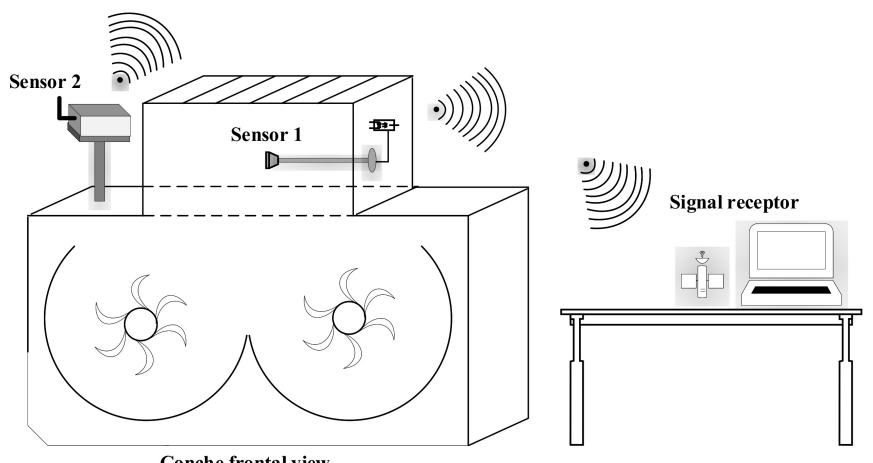

Fig. 2. Location of the temperature and moisture sensors.

To formulate the output of the system, a new process system $P S_{I I I}$ corresponding to the upper conche compartment is considered (Figure 3). Additionally, to directly relate the measure from sensor 1, a mass balance of water in the upper conche compartment is formulated as:

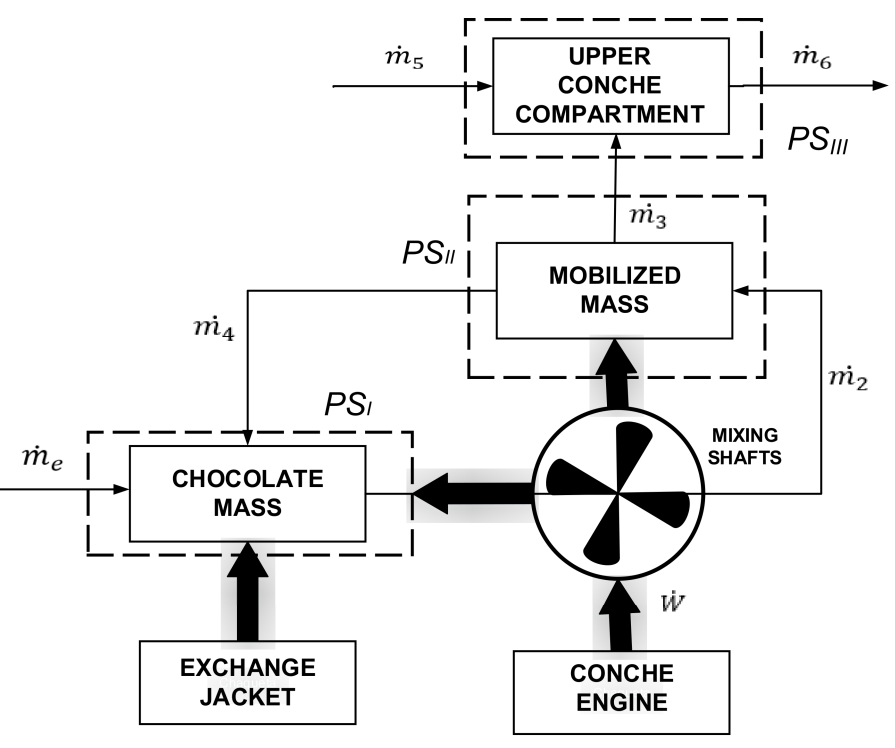

Fig. 3. Process Systems for the conching process

$$
\frac{d}{d t}\left(M_{c} w_{H_{2} O, 6}\right)=\dot{m}_{3} w_{H_{2} O, 3}+\dot{m}_{5} w_{H_{2} O, 5}-\dot{m}_{6} w_{H_{2} O, 6},
$$

where $w_{H_{2} O, 5}$ is the absolute moisture in the sourronding air $\left[\mathrm{kg}_{\mathrm{H}_{2} \mathrm{O}} / \mathrm{kg}_{\mathrm{AS}}\right]$, obtained using the relative moisture measure from sensor 2, $w_{H_{2} O, 6}$ corresponds to the absolute moisture content in the upper conche compartment $\left[\mathrm{kg}_{\mathrm{H}_{2} \mathrm{O}} / \mathrm{kg}_{\mathrm{AS}}\right]$ obtained by using the relative moisture measure from sensor 1, $\dot{m}_{5}$ and $\dot{m}_{6}$ are the mass flow of dry air in the inlet and outlet, respectively, of the upper conche compartment $\left[\mathrm{kg}_{\mathrm{H}_{2} \mathrm{O}} / \mathrm{min}\right]$. Due to the lack of information of these inlet and outlet mass flows, $\dot{m}_{5}$ and $\dot{m}_{6}$ were identified using the real data obtained from sensors 1 and 2. Finally, $M_{c}$ is the air mass in the upper conche compartment $[\mathrm{kg}]$, calculated considering its volume , i.e,

$$
M_{c}=\rho_{\text {air }} L_{c}^{3},
$$

where $L_{c}$ is the length side of the compartment $[\mathrm{m}]$ and $\rho_{\text {air }}$ is the air density $\left[\mathrm{kg} / \mathrm{m}^{3}\right]$.

Considering the new balance (14) as a part of the ODE system (13), it is possible to obtain $w_{\mathrm{H}_{2} \mathrm{O}, 6}$ as an additional differential variable $x_{a}$. Then, analyzing the ODE system, a classification of the variables is presented in Table III. Note that there are 18 state variables from which two of them are measured.

Finally, considering the system's output function as a part of the model structure in (13), the resulting model is written as follows:

$$
\left\{\begin{array}{l}
\dot{x}_{a}=F_{a}\left(t, x_{a}, u\right) \\
y_{1}=T \\
y_{2}=w_{H_{2} O, 6}
\end{array}\right.
$$


TABLE III

CLASSIFICATION OF THE ODE SYSTEM VARIABLES

\begin{tabular}{l|l}
\hline Type & Symbol \\
\hline \hline State variables & $x_{a}=\left[M_{P S_{I}}, w_{H_{2} O, j}, w_{B A, j} w_{T M P, j}, w_{e f}, T\right.$, \\
Measured outlets & $\left.\omega_{s}, \dot{m}_{4}, Q, P_{c}, N_{R e}, \mu, \sigma, \gamma, w_{H_{2} O, 6}\right]^{T}$ \\
& $y=\left[T, w_{H_{2} O, 6}\right]^{T}$ \\
\hline
\end{tabular}

with $j \in[2,4]$

\section{B. Local observability analysis}

At this point, observability analysis can be performed. According to the definition of local observability, it is necessary to find some equilibrium point of the system, namely $x_{a}^{*}$. Taking into account that the conching process is analyzed as a batch process, the observability analysis is performed for the model at each time instant $\left(t^{*}, x_{a}^{*}\right)$ corresponding to the solution of the nonlinear model at each point $\left(t^{*}\right)$ defined in the nominal trajectory of the system.

Using the non-linear model shown in (16), the model is linearized by computing matrices $A, B$ and $C$ at each point $\left(t^{*}, x_{a}^{*}\right)$ of the nominal trajectory, i.e.,

$$
\begin{aligned}
\Delta \dot{x_{a}} & =A \Delta x_{a}+B \Delta u \\
\Delta y & =C \Delta x_{a}
\end{aligned}
$$

with $\Delta x_{a}=x_{a}-x_{a}^{*}, \Delta u=u-u^{*}, \Delta y=y-y^{*}$ being the deviation variables for the states, the known inputs, and the outputs, respectively, while $A=\left.\frac{\partial F_{a}}{\partial x_{a}}\right|_{\left(t^{*}, x_{a}^{*}\right)}$, $B=\left.\frac{\partial F_{a}}{\partial u}\right|_{\left(t^{*}, x_{a}^{*}\right)}$ and $C=\left.\frac{\partial h}{\partial x_{a}}\right|_{\left(t^{*}, x_{a}^{*}\right)}$.

Taking into account that the observability relates the system states with its outputs, the observability matrix $M_{o}$ is calculated for as follows each time instant $\left(t^{*}, x_{a}^{*}\right)$ considering the matrices $A$ and $C$ :

$$
\left.M_{o}\right|_{\left(t^{*}, x_{a}^{*}\right)}=\left[\begin{array}{c}
C \\
C A \\
C A^{2} \\
\cdot \\
\cdot \\
C A^{(n-1)},
\end{array}\right]
$$

with $n$ the number of states, $A_{(18 \times 18)}$ and $C_{(18 \times 2)}$ matrices evaluated at time instant $\left(t^{*}, x_{a}^{*}\right)$. Finally, the system is observable for each point $\left(t^{*}, x_{a}^{*}\right)$ if and only if $\operatorname{Rank}\left(M_{o}\right)=n$ where $\mathrm{n}$ is the dimension of the state vector (in this case $n=18$ ).

When performing the analysis for all points $\left(t^{*}, x_{a}^{*}\right)$ of the nominal trajectory of the model, it was found that for the observability matrix in (18) $\operatorname{Rank}\left(M_{o}\right)=7$ which means that some states are re-constructable (observable) from the output, but their measurements are not enough to reconstruct some other state variables.

However, while observability is a sufficient condition for the estimation of process state variables, there is a necessary and sufficient notion for the estimation called detectability. The concept of detectability is close to observability. A system is detectable if and only if all unstable modes are observable. That is, when the state vector of a system is not completely re-constructable, but the state that is not re-constructable converges to a steady state value, the system is detectable.

In that sense, taking into account the notion of detectability and evaluating the system's stability by the analysis of the eigenvalues, $\lambda_{i}$, of matrix $\mathrm{A}$ at the end of the batch $\left(\lambda_{i} \in\right.$ $\left.\left(-40.6,-6.3 \times 10^{-16}\right) \in \mathbb{R}^{-}\right)$, the system is defined as stable and therefore detectable for each point $\left(t^{*}, x_{a}^{*}\right)$

\section{STATE ESTIMATOR PROPOSAL}

A proposal of a Nonlinear Moving Horizon Estimator (NLMHE) to estimate the CD $[\mathrm{mg} / \mathrm{kg}]$ and the chocolate viscosity $\mu\left[\mathrm{Pa}^{*} \mathrm{~s}\right]$ by using the non-linear model presented in (16) is proposed. It should be noted that since the CD is not a state variable of the process, the knowledge of the estimation of two state process variables, i.e, the concentration of BA $w_{B A, 4}\left[\mathrm{mg} B A / \mathrm{kg}_{\mathrm{DS}}\right]$ and the concentration of TMP $w_{T M P, 4}\left[\mathrm{mg} T M P / \mathrm{kg}_{\mathrm{DS}}\right]$ is required, as stated in (1). The estimation structure of the proposed NLMHE is sketched in Figure 4, where the estimator requires the inputs and outputs of the conching process model, which represent the real process. With the proposed structure, the TMP concentration $w_{T M P, 4}$, and the BA concentration $w_{B A, 4}$ can be observed directly by the NLMHE. Moreover, since $\mu$ is a state variable of the ODE system proposed in Section II, its observation can be also obtained directly using the NLMHE. Then, observed TMP and BA concentrations will be used for internal calculation of the estimated $\mathrm{CD}$ as well as the inputs and outputs of the conching process model by a block calculation.

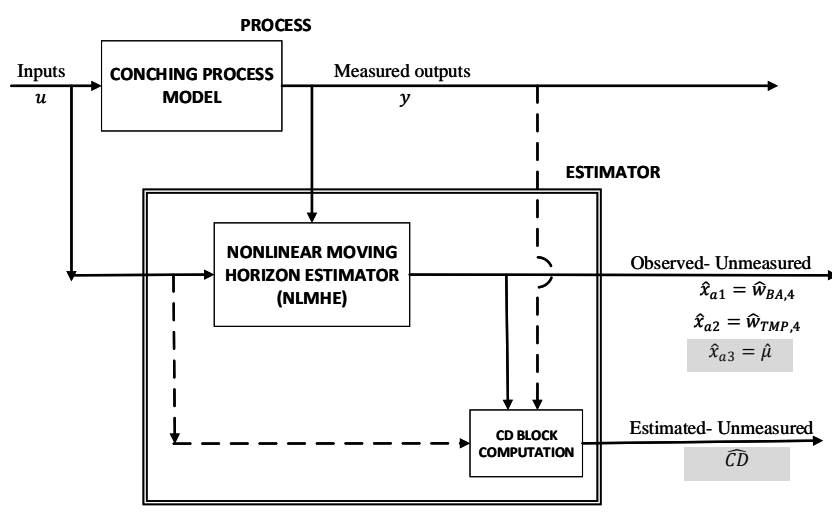

Fig. 4. Estimation structure using an NLMHE for the estimation of CD and chocolate viscosity.

\section{A. Simulation results}

Some preliminary results are shown in Figure 5, where the comparison among the estimation of the measured variables, i.e., chocolate temperature and moisture content in the upper 
conche's compartment, are shown. According to Figure 5, the proposed estimator follows the real behavior with small differences between the value of the real variable and the estimation. The obtained estimation has a suitable noise propagation and the estimated value follows smoothly the real value of the variable.
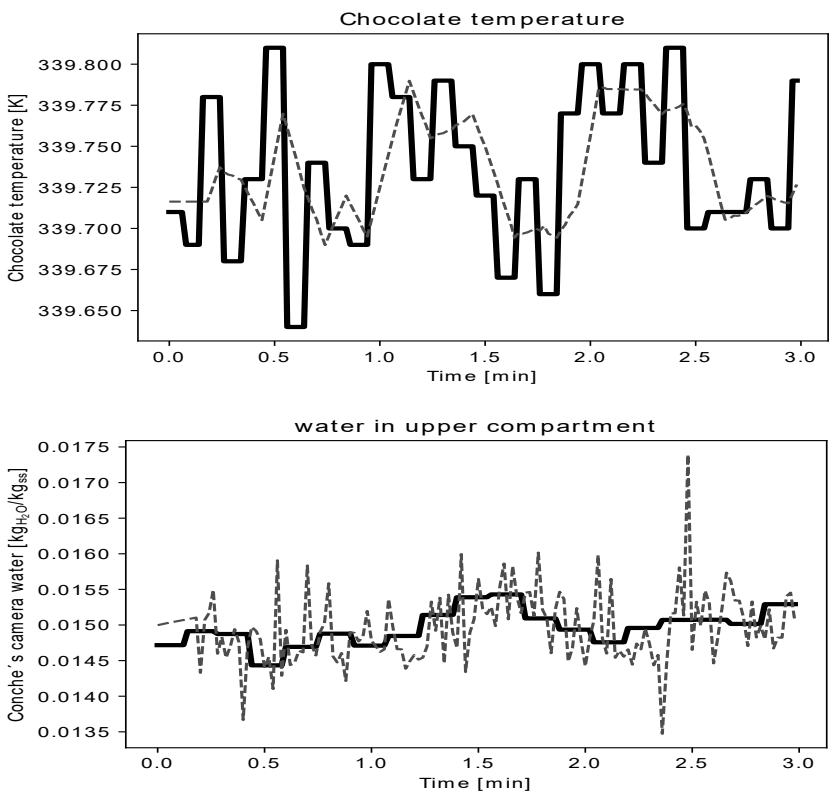

Fig. 5. Estimation of the measured variables, i.e., $T$ and $w_{H 2 O, 6}$ from 0 to $3 \mathrm{~min}$, where the black continuous lines correspond to the real value of the variable and the gray dotted lines correspond to its estimated value

\section{Conclusions}

A proposal of virtual sensor to predict the Conching Degree and the viscosity of a chocolate batch was presented. To this end, the conditions of detectability for the estimator design were satisfied by the selection of the state variables, the known inputs, the outputs, and the equations considered in the model structure. In addition, an estimation structure where the TMP concentration, the BA concentration, and chocolate's viscosity were estimated is proposed, considering only two measured variables available from the process. Thus, by the knowledge of the estimation of TMP and the BA concentrations, it is possible to estimate indirectly the $\mathrm{CD}$ of the chocolate. Some preliminary results show that the state estimator proposal has a suitable performance. The complete design of the virtual sensor and the real implementation are considered as future work.

\section{ACKNOWLEDGMENT}

This work is financed by the project supported by Compañia Nacional de Chocolates and COLCIENCIAS 786-2017 "Beneficios Tributarios por inversión - CTeI".

\section{REFERENCES}

[1] G. Ziegleder, "Fat migration," Fraunhofer Institute for Processing and Packaging, Tech. Rep., 2004.

[2] E. O. Afoakwa, Chocolate Science and Technology. John Wiley Sons, Ltd, 2011.

[3] W. Danzl and G. Ziegleder, "Chocolate conching: Aroma improvement by a changed flavour distribution," Freising, Germany, p. 9, 2014.

[4] H. Botero and H. Alvarez, "La Estimación Del Estado En Procesos Químicos a Revision of the Most Frequent Methods for State Estimation in Chemical Processes," Dyna, vol. 72, pp. 135-146, 2009.

[5] J. Oliveira, J. N. Santos, and P. Seleghim, "Inverse measurement method for detecting bubbles in a fluidized bed reactor toward the development of an intelligent temperature sensor," Powder Technology, vol. 169, no. 3, pp. 123-135, 2006.

[6] J. Tan and W. L. Kerr, "Determining degree of roasting in cocoa beans by Artificial Neural Network (ANN) based electronic nose system and Gas Chromatography Mass Espectrometry (GC-MS)," Journal of the Science of Food and Agriculture, vol. 98, p. 10, 2018.

[7] — , "Characterizing cocoa refining by electronic nose using a Kernel distribution model," LWT - Food Science and Technology, vol. 104, no. October 2018, pp. 1-7, 2019. [Online]. Available: https://doi.org/10.1016/j.lwt.2019.01.028

[8] J. Tan and B. M. Balasubramanian, "Particle size measurements and scanning electron microscopy (SEM) of cocoa particles refined / conched by conical and cylindrical roller stone melangers," Journal of Food Engineering, vol. 212, pp. 146-153, 2017.

[9] C. González, E. V. Acosta, D. A. Muñoz, J. Rúa Torres, W. Valencia Gallego, and J. C. Mazo Rivas, "Phenomenological model for predicting the Conching Degree of chocolate," Journal of Food Engineering, vol. 289, p. 110147, 2021.

[10] C. González, E. V. Acosta, J. C. Mazo Rivas, and D. A. Muñoz, "Phenomenological based model for the prediction of the structural changes during chocolate conching process," Journal of Food Engineering, vol. 289, p. 110184, 2021.

[11] M. K. Ramalingam, "Moving Horizon Estimation with Dynamic Programming," Ph.D. dissertation, Cleveland State University, 2013.

[12] S. T. Beckett, The Science of chocolate. The Royal Society of Chemistry, 2008. 